\title{
Does Spatial Navigation Have a Blind-Spot? Visiocentrism Is Not Enough to Explain the Navigational Behavior Comprehensively
}

\author{
Mateusz Hohol ${ }^{1,2}$, Bartosz Baran ${ }^{3}$, Michał Krzyżowski ${ }^{3}$ and Jacek Francikowski ${ }^{3 *}$ \\ ${ }^{1}$ Department of Logic and Cognitive Science, Institute of Philosophy and Sociology, Polish Academy of Sciences, Warsaw, \\ Poland, ${ }^{2}$ Copernicus Center for Interdisciplinary Studies, Kraków, Poland, ${ }^{3}$ Department of Animal Physiology and \\ Ecotoxicology, Faculty of Biology and Environmental Protection, University of Silesia, Katowice, Poland
}

Keywords: spatial navigation, geometric module, view-matching, insect studies, multimodal integration

OPEN ACCESS

Edited by:

Bruno Poucet

Centre National De La Recherche Scientifique (CNRS), France

Reviewed by: Antoine Wystrach, UMR5169 Centre De Recherches Sur La Cognition Animale (CRCA), France

*Correspondence: Jacek Francikowski jacek.francikowski@us.edu.pl

Received: 05 July 2017 Accepted: 04 August 2017 Published: 18 August 2017

Citation:

Hohol M, Baran B, Krzyżowski M and

Francikowski J (2017) Does Spatial Navigation Have a Blind-Spot?

Visiocentrism is Not Enough to Explain the Navigational Behavior

Comprehensively.

Front. Behav. Neurosci. 11:154. doi: 10.3389/fnbeh.2017.00154
A few years ago Wystrach and Graham (2012b) asked: "What can we learn from studies of insect navigation?" They identified that complex navigational behavior of insects can be explained via fairly simple mechanisms, such as view-matching (Wehner and Räber, 1979; Cartwright and Collett, 1983), without referring to the high-level mental mechanisms (Cheng, 1986). Furthermore, they suggested that since the navigational behavior of vertebrates, show significant convergence with insects' behavior, it is justified to consider mechanism based on egocentric views before assuming the existence of higher-level mechanisms.

Such a point of view seems to fall in line with a broader consensus, and wider paradigm shift that has taken place in recent years. For instance, Cheng (2008), the former proponent of the high-level geometric module, suggested that view-matching models of insects' navigation, can be directly implemented in the studies on the spatial navigation of vertebrates, and even human. This paradigm-shift was also driven by successes in implementing view-matching to behavioral robotics (Möller and Vardy, 2006).

To test for the use of egocentric view (i.e., view based matching hypotheses), most studies use pixel-by-pixel image comparison, and some results in both vertebrates and insects reject the prediction made by such image comparison (Lehrer et al., 1988; Lee et al., 2012). However, it is argued that rejecting pixel-by-pixel comparison is not rejecting the use of view-based matching mechanisms, as animal views are not "images" but can encode parameters such as depths, motion, edges or specific features, which varies across species (Wystrach and Graham, 2012a). In this light, pixel-by-pixel models of insect navigation seem unrealistic and should not be understood literally, but rather as a proxy for quantifying surroundings familiarity. However, and beyond that debate, it is clear that reorientation can be accomplished in ways excluding any type of view-based-matching mechanism, such as when accomplished by a blindfolded human (Sturz et al., 2013). Furthermore, Cheng et al. (2013) pointed out that in the contemporary literature on spatial navigation there are several competing approaches, and the field lacks a unified research paradigm.

In this paper, we argue that the issues described arise not because of the lack of theoretical inspiration, but rather due to an insufficient understanding of the subtleties of insect behavior. In our view, implementation of the insects' models of navigation in the explanation of the vertebrates' spatial behavior omits some important aspects, i.e., multimodal integration. Thus, we want to ask again the initial question posed by Wystrach and Graham (2012b) pointing out that significant progress in insects' research, which suggests that we might have had underestimated insects' cognitive abilities (Loukola et al., 2017; Peng and Chittka, 2017). Those results demonstrated insects' capacity to obtain abstract information from multimodal input during complex tasks. Movement through a real environment provides a variety of cues, not only visual ones, thus in the following article we argue that multimodal integration is crucial to navigation. 


\section{VISIOCENTRISM IN THE STUDIES ON SPATIAL NAVIGATION}

Vertebrates' capacity for spatial navigation has been traditionally perceived as the product of the activity of the so-called "geometric module" (Fodor, 1983), which encodes only the geometric shape of the environment via purely visual input (Gallistel, 1990). This thesis was derived primarily from the results of behavioral studies (Cheng, 1986), in which familiarized animals after a disorientation phase, searched for a reward hidden in a given corner of a rectangular arena, relying mainly on the visually perceived geometry of the apparatus (see Thinus-Blanc et al., 2010 for review). The geometric module was thought to be localized in the hippocampus (Vargas et al., 2004) and separated from both domain-general processes as well as other domainspecific cognitive modules (Gallistel, 1990).

The further studies conducted with various species, especially human (Hermer and Spelke, 1994) and non-human primates (Gouteux et al., 2001), have showed that vertebrates' spatial navigation sometimes depends on the productive combination of geometric and non-geometric information. Namely animals can optimize task performance by using recognized featural cues or landmarks. These results have triggered conceptual shift. According to Spelke et al. (2010), spatial navigation depends on two "core cognitive systems" for processing three-dimensional spatial layouts and two-dimensional visual forms respectively.

Spatial navigation is, however, still thought to be a product of high-level mechanisms which process information retrieved from visual input. Even earlier attempts (Cheung et al., 2008) to implement a view-matching approach (Wehner and Räber, 1979; Wehner, 2003) to vertebrate's spatial navigation studies haven't changed the research perspective as radically as it seemed. The tendency to focus on solely visual-driven mechanisms in studies of spatial navigation, with simultaneous neglect of other modalities, we have decided to refer to as: visiocentrism. It seems that the visiocentric perspective still dominates the study of insect and vertebrate navigation.

In the following sections, we aim to challenge this viewpoint from evolutionary, neurophysiological and behavioral perspectives. We point out that the current state of understanding of the evolutionary economics seems incoherent with visiocentric models of navigation, in terms of insect nervous systems and information processing. Furthermore, we discuss evidence from behavioral studies utilizing non-visual/mixed approaches, to support our claim that multimodal integration plays crucial role in spatial navigation.

\section{SPATIAL NAVIGATION IN LITTLE BRAINS}

During phylogenesis, nervous systems tend to undergo specialization in order to achieve fitness to the ecological niche of a particular species (Liebeskind et al., 2016). Despite divergence of the evolutionary pathways of arthropods and vertebrates, many authors point out functional and structural similarities in their visual systems, specifically in neural circuits specialized for visual processing (Borst and Euler, 2011; Shih et al., 2015). The most important feature of all these systems is an economy of neuronal wiring (Chen et al., 2006). This principle is observed on retina level (neuronal convergence) and in the central nervous system (Laughlin et al., 1998). Basic restrictions of nervous system complexity are the size of the head cavity, and high energy demand, as neuronal conduction of information on the axonal and synaptic level is very energy-intensive (Laughlin et al., 1998). By understanding the neuronal economy, the functional economy can be postulated. The amount of information which can be transmitted through the nervous system in the unit of time is restricted by many factors (Bullmore and Sporns, 2012). The solution to this problem is to, at very early stage, select, reduce and compress information (Nityananda, 2016).

The second important mechanism used to deal with excess information in the nervous system, is the set of models and algorithms (Wehner, 1987; Webb, 2004). This clearly demonstrates that authentic reproduction of the external environment is impossible, and brains create a simplified representation of an environment with emphasis on key elements (Doyle and Csete, 2011). Visual information on the level of optical lobes is not analyzed as a unitary structure but is divided on movement reception, related to optomotor response, and object features such as color, small object movement, and shape (Dunbier et al., 2012). We know that, in insects, sensory information is analyzed in small loops specialized in the preliminary analysis, known as the small-world network. Subsequently, information flow is redirected to main associative structures: mushroom bodies (MBs) and central complex (CC) (Strausfeld et al., 1998). It is uncommon for these structures be connected directly by afferent or efferent pathways, whether sensory or effectory. Additionally, visual information does not remain separate but is coupled with other modalities (Borst and Euler, 2011). The MBs and CC are structures with numerous outputs and inputs of various modalities, and thus are considered as a higher-order multimodal information integration centers (Wessnitzer and Webb, 2006; Avargues-Weber and Giurfa, 2013; Giurfa, 2013).

Depending on evolutionary pressures, neuronal background can differ between insect species (Wehner, 1987). Nevertheless, presence of MBs and CB is universal in insecta class and, as it was stated above, those structures play crucial role in spatial memory and spatial navigation. While it seems counterintuitive that units described as sensory afferents can carry more than one modality, intracellular recordings have revealed that many afferents to both structures (MBs and CC) are multimodal (Li and Strausfeld, 1997). This suggests that higher processing instances does not have access to raw sensory information, and locomotion cannot be regulated directly by simple activity of retina cells. Additionally, during transmission, visual information from eyes undergoes intensive processing (Zipursky and Sanes, 2010). In all insects, single neurons connecting optic lobes with higher associative structures can be observed. This feature can be seen as a manifestation of economical wiring. These neurons originate in areas that receive collaterals and terminals of visual interneurons, from the ipsilateral and contralateral optic lobes, providing consistent information flow (Li and Strausfeld, 1997; Mizunami et al., 1998). Connections between small local 
loops are usually weaker (contain fewer neurons) than internal connections. It can be an advantage for noise-limited signaling systems. A low capacity pathway (fewer neurons) transmits information in a more economically efficient way, providing better distribution of information among different pathways (Laughlin et al., 1998). The main function of nervous systems is extract and track environmental regularities. Knowledge of these regularities is utilized to anticipate the consequences of movements, thus allowing the most adaptive action selection (Webb, 2012). Those regularities span, however, across various modalities, which appears to be reflected in organization of insects' brains. Therefore, we claim that the visiocentric approach neglects crucial aspects of insects' brain anatomy.

\section{BEYOND VISIOCENTRISM}

In recent decades, we can observe a change in the approach to insect navigation research. The vision-based strategies, especially the view-matching, has been gaining more and more attention (Wystrach and Graham, 2012a; Avargues-Weber and Giurfa, 2013). The discovery of mammalian-like rotational errors of ants navigating in rectangular arenas (Wystrach and Beugnon, 2009) and the success of view based matching in explaining these results (Stürzl et al., 2008; Wystrach et al., 2011) led to the dissemination of these view-matching models in the vertebrate literature, and, as a corollary, strengthened the unjustified perception of insects as rather simplistic, yet highly adapted agents.

The described shift caused the emergence of visiocentrism, however current data on a neuronal foundation of navigational processes strongly suggest that this approach may be incomplete. According to the arguments already presented and to recently developed models (Webb and Wystrach, 2016; Roper et al., 2017), the insect's brain possesses architecture adapted for sensory integration rather than processing single modalities separately. This data is coherent with observations concerning the use of non-visual or combined cues in insects (Chittka et al., 1999; Thiélin-Bescond and Beugnon, 2005; Wystrach and Schwarz, 2013; Buehlmann et al., 2015; Minoura et al., 2016; Raderschall et al., 2016).

Gallistel (1990) argued that the geometric module is evolutionarily justified, since geometric information remains stable in contrary to changes of the features. A similar argument may be formulated for multimodal integration. When various modalities are considered, chances of misrecognition of object or place are much lower. Even if single modality featural cues change, chances are that in other modalities features may remain:

\section{REFERENCES}

Avargues-Weber, A., and Giurfa, M. (2013). Conceptual learning by miniature brains. Proc. R. Soc. B Biol. Sci. 280:20131907. doi: 10.1098/rspb. 2013.1907

Borst, A., and Euler, T. (2011). Seeing things in motion: models, circuits, and mechanisms. Neuron 71, 974-994. doi: 10.1016/j.neuron.2011. 08.031 food will retain its smell and taste even if it is smashed or cut. The visual system is one of the most energetically demanding and structurally elaborated systems in the brain (Wong-Riley, 2010), therefore managing computationally complex tasks using only visual instances of the brain seems evolutionary uneconomical. Visual information may be supplemented by other senses to provide more reliable information about surroundings, simultaneously reducing metabolic cost.

Likewise, vertebrates' hippocampi, like the $\mathrm{CB}$ and $\mathrm{MBs}$ of insects, are provided with inputs from all sensory modalities (Ravassard et al., 2013). These structures are known to be crucial in navigational processes (Mizunami et al., 1998; Pfeiffer and Homberg, 2014), and therefore it is hardly a surprise that senses other than vision are utilized during navigation. Even without vision, blind rats could successfully accomplish navigation if place cells were functioning correctly (Save et al., 1998). In the case of mammalian navigation, there is direct evidence of multimodal input role in the function of place cells (Ravassard et al., 2013). Although, what is surprising, is that the studies investigating reorientation almost entirely neglect this aspect (Cheng, 2008) despite the known role of multimodal integration in perception (Cheng et al., 2007). Therefore, we claim that it would be beneficial for studies on spatial navigation, both in invertebrates and vertebrates, to expand perspective further and, include non-visual modalities. This perspective may be of great value, especially when questions considering the internal representation of space are asked.

\section{AUTHOR CONTRIBUTIONS}

$\mathrm{MH}, \mathrm{BB}, \mathrm{MK}$, and JF reviewed the literature and developed the theoretical stance. $\mathrm{MH}, \mathrm{BB}, \mathrm{MK}$, and JF wrote the manuscript. $\mathrm{MH}, \mathrm{BB}, \mathrm{MK}$, and JF reviewed and accepted its final version.

\section{FUNDING}

Preparation of the manuscript was supported by the research grant 2015/19/B/HS1/03310 "Mechanisms of geometric cognition" funded by National Science Centre, Poland.

\section{ACKNOWLEDGMENTS}

We would like to thank Dr. Antoine Wystrach who provided many helpful comments on the previous version of the manuscript. 
Chen, B. L., Hall, D. H., and Chklovskii, D. B. (2006). Wiring optimization can relate neuronal structure and function. Proc. Natl. Acad. Sci. U.S.A. 103, 4723-4728. doi: 10.1073/pnas.0506806103

Cheng, K. (1986). A purely geometric module in the rat's spatial representation. Cognition 23, 149-178. doi: 10.1016/0010-0277(86)90041-7

Cheng, K. (2008). Whither geometry? Troubles of the geometric module. Trends Cogn. Sci. 12, 355-361. doi: 10.1016/j.tics.2008.06.004

Cheng, K., Huttenlocher, J., and Newcombe, N. S. (2013). 25 years of research on the use of geometry in spatial reorientation: a current theoretical perspective. Psychon. Bull. Rev. 20, 1033-1054. doi: 10.3758/s13423-013-0416-1

Cheng, K., Shettleworth, S. J., Huttenlocher, J., and Rieser, J. J. (2007). Bayesian integration of spatial information. Psychol. Bull. 133, 625-637. doi: 10.1037/0033-2909.133.4.625

Cheung, A., Stürzl, W., Zeil, J., and Cheng, K. (2008). The information content of panoramic images II: view-based navigation in nonrectangular experimental arenas. J. Exp. Psychol. Anim. Behav. Process. 34, 15-30. doi: 10.1037/0097-7403.34.1.15

Chittka, L., Williams, N. M., Rasmussen, H., and Thomson, J. D. (1999). Navigation without vision: bumblebee orientation in complete darkness. Proc. R. Soc. B Biol. Sci. 266, 45-50. doi: 10.1098/rspb.1999.0602

Doyle, J. C., and Csete, M. (2011). Architecture, constraints, and behavior. Proc. Natl. Acad. Sci. U.S.A. 108, 15624-15630. doi: 10.1073/pnas.11035 57108

Dunbier, J. R., Wiederman, S. D., Shoemaker, P. A., and O'Carroll, D. C. (2012). Facilitation of dragonfly target-detecting neurons by slow moving features on continuous paths. Front. Neural Circuits 6:79. doi: 10.3389/fncir.2012. 00079

Fodor, J. A. (1983). The Modularity of Mind. Cambridge, MA: The MIT Press.

Gallistel, C. (1990). The Organization of Learning. Cambridge, MA: The MIT Press.

Giurfa, M. (2013). Cognition with few neurons: higher-order learning in insects. Trends Neurosci. 36, 285-294. doi: 10.1016/j.tins.2012.12.011

Gouteux, S., Thinus-Blanc, C., and Vauclair, J. (2001). Rhesus monkeys use geometric and nongeometric information during a reorientation task. J. Exp. Psychol. Gen. 130, 505-519. doi: 10.1037/0096-3445.130.3.505

Hermer, L., and Spelke, E. S. (1994). A geometric process for spatial orientation in young children. Nature 370, 57-59. doi: 10.1038/370057a0

Laughlin, S. B., van Steveninck, R., and Anderson, J. C. (1998). The metabolic cost of neural information. Nat. Neurosci. 1, 36-41. doi: 10.1038/236

Lee, S. A., Spelke, E. S., and Vallortigara, G. (2012). Chicks, like children, spontaneously reorient by three-dimensional environmental geometry, not by image matching. Biol. Lett. 8, 492-494. doi: 10.1098/rsbl.2012.0067

Lehrer, M., Srinivasan, M. V., Zhang, S. W., and Horridge, G. A. (1988). Motion cues provide the bee's visual world with a third dimension. Nature 332, 356-357. doi: 10.1038/332356a0

Li, Y., and Strausfeld, N. J. (1997). Morphology and sensory modality of mushroom body extrinsic neurons in the brain of the cockroach, Periplaneta americana. J. Comp. Neurol. 387, 631-650. doi: 10.1002/(SICI)1096-9861 (19971103)387:4<631::AID-CNE9>3.0.CO;2-3

Liebeskind, B. J., Hillis, D. M., Zakon, H. H., and Hofmann, H. A. (2016). Complex homology and the evolution of nervous systems. Trends Ecol. Evol. 31, 127-135. doi: 10.1016/j.tree.2015.12.005

Loukola, O. J., Perry, C. J., Coscos, L., and Chittka, L. (2017). Bumblebees show cognitive flexibility by improving on an observed complex behavior. Science 355, 833-836. doi: 10.1126/science.aag2360

Minoura, M., Sonoda, K., Sakiyama, T., and Gunji, Y.-P. (2016). Rotating panoramic view: interaction between visual and olfactory cues in ants. $R$. Soc. Open Sci. 3:150426. doi: 10.1098/rsos.150426

Mizunami, M., Weibrecht, J. M., and Strausfeld, N. J. (1998). Mushroom bodies of the cockroach: their participation in place memory. J. Comp. Neurol. 402, 520-537. doi: 10.1002/(SICI)1096-9861(19981228) 402:4<520::AID-CNE6>3.0.CO;2-K

Möller, R., and Vardy, A. (2006). Local visual homing by matched-filter descent in image distances. Biol. Cybern. 95, 413-430. doi: 10.1007/s00422-0060095-3

Nityananda, V. (2016). Attention-like processes in insects. Proc. R. Soc. B Biol. Sci. 283:20161986. doi: 10.1098/rspb.2016.1986

Peng, F., and Chittka, L. (2017). A simple computational model of the bee mushroom body can explain seemingly complex forms of olfactory learning and memory. Curr. Biol. 27, 224-230. doi: 10.1016/j.cub.2016. 10.054

Pfeiffer, K., and Homberg, U. (2014). Organization and functional roles of the central complex in the insect brain. Annu. Rev. Entomol. 59, 165-184. doi: 10.1146/annurev-ento-011613-162031

Raderschall, C. A., Narendra, A., and Zeil, J. (2016). Head roll stabilisation in the nocturnal bull ant Myrmecia pyriformis: implications for visual navigation. J. Exp. Biol. 219, 1449-1457. doi: 10.1242/jeb.134049

Ravassard, P., Kees, A., Willers, B., Ho, D., Aharoni, D., Cushman, J., et al. (2013). Multisensory control of hippocampal spatiotemporal selectivity. Science 340, 1342-1346. doi: 10.1126/science. 1232655

Roper, M., Fernando, C., and Chittka, L. (2017). Insect bio-inspired neural network provides new evidence on how simple feature detectors can enable complex visual generalization and stimulus location invariance in the miniature brain of honeybees. PLoS Comput. Biol. 13:e1005333. doi: 10.1371/journal.pcbi.10 05333

Save, E., Cressant, A., Thinus-Blanc, C., and Poucet, B. (1998). Spatial firing of hippocampal place cells in blind rats. J. Neurosci. 18, 1818-1826.

Shih, C.-T., Sporns, O., Yuan, S.-L., Su, T.-S., Lin, Y.-J., Chuang, C.-C., et al. (2015). Connectomics-based analysis of information flow in the drosophila brain. Curr. Biol. 25, 1249-1258. doi: 10.1016/j.cub.2015.03.021

Spelke, E. S., Lee, S. A., and Izard, V. (2010). Beyond core knowledge: natural geometry. Cogn. Sci. 34, 863-884. doi: 10.1111/j.1551-6709.2010.01110.x

Strausfeld, N. J., Hansen, L., Li, Y., Gomez, R. S., and Ito, K. (1998). Evolution, discovery, and interpretations of arthropod mushroom bodies. Learn. Mem. 5, 11-37. doi: 10.1101/lm.5.1.11

Sturz, B. R., Green, M. L., Gaskin, K. A., Evans, A. C., Graves, A. A., and Roberts, J. E. (2013). More than a feeling: incidental learning of array geometry by blindfolded adult humans revealed through touch. J. Exp. Biol. 216, 587-593. doi: 10.1242/jeb.080952

Stürzl, W., Cheung, A., Cheng, K., and Zeil, J. (2008). The information content of panoramic images I: the rotational errors and the similarity of views in rectangular experimental arenas. J. Exp. Psychol. Anim. Behav. Process. 34, 1-14. doi: 10.1037/0097-7403.34.1.1

Thiélin-Bescond, M., and Beugnon, G. (2005). Vision-independent odometry in the ant Cataglyphis cursor. Naturwissenschaften 92, 193-197. doi: 10.1007/s00114-005-0609-1

Thinus-Blanc, C., Chabanne, V., Tomassi, L., Peruch, P., and Vauclair, J. (2010). "The encoding of geometry in various vertebrate species," in Spatial Cognition, Spatial Perception, eds F. L. Dolins and R. W. Mitchell (Cambridge: Cambridge University Press), 99-116.

Vargas, J. P., Petruso, E. J., and Bingman, V. P. (2004). Hippocampal formation is required for geometric navigation in pigeons. Eur. J. Neurosci. 20, 1937-1944. doi: 10.1111/j.1460-9568.2004.03654.x

Webb, B. (2004). Neural mechanisms for prediction: do insects have forward models? Trends Neurosci. 27, 278-282. doi: 10.1016/j.tins.2004.03.004

Webb, B. (2012). Cognition in insects. Philos. Trans. R. Soc. B Biol. Sci. 367, 2715-2722. doi: 10.1098/rstb.2012.0218

Webb, B., and Wystrach, A. (2016). Neural mechanisms of insect navigation. Curr. Opin. Insect Sci. 15, 27-39. doi: 10.1016/j.cois.2016.02.011

Wehner, R. (1987). "Matched filters": neural models of the external world. J. Comp. Physiol. Sens. Neural Behav. Phys. 161, 511-531. doi: 10.1007/BF00603659

Wehner, R. (2003). Desert ant navigation: how miniature brains solve complex tasks. J. Comp. Phys. Sens. Neural and Behav. Phys. 189, 579-588. doi: 10.1007/s00359-003-0431-1

Wehner, R., and Räber, F. (1979). Visual spatial memory in desert ants, Cataglyphis bicolor (Hymenoptera: Formicidae). Experientia 35, 1569-1571. doi: 10.1007/BF01953197

Wessnitzer, J., and Webb, B. (2006). Multimodal sensory integration in insects: towards insect brain control architectures. Bioinspir. Biomim. 1, 63-75. doi: 10.1088/1748-3182/1/3/001

Wong-Riley, M. (2010). Energy metabolism of the visual system. Eye Brain 2:99. doi: $10.2147 /$ EB.S9078

Wystrach, A., and Beugnon, G. (2009). Ants learn geometry and features. Curr. Biol. 19, 61-66. doi: 10.1016/j.cub.2008.11.054

Wystrach, A., and Graham, P. (2012a). View-based matching can be more than image matching: the importance of considering an animal's perspective. $i$ Perception 3, 547-549. doi: 10.1068/i0542ic 
Wystrach, A., and Graham, P. (2012b). What can we learn from studies of insect navigation? Anim. Behav. 84, 13-20. doi: 10.1016/j.anbehav.2012.04.017

Wystrach, A., and Schwarz, S. (2013). Ants use a predictive mechanism to compensate for passive displacements by wind. Curr. Biol. 23, R1083-R1085. doi: 10.1016/j.cub.2013.10.072

Wystrach, A., Cheng, K., Sosa, S., and Beugnon, G. (2011). Geometry, features, and panoramic views: ants in rectangular arenas. J. Exp. Psychol. Anim. Behav. Process. 37, 420-435. doi: 10.1037/a0023886

Zipursky, S. L., and Sanes, J. R. (2010). Chemoaffinity revisited: dscams, protocadherins, and neural circuit assembly. Cell 143, 343-353. doi: 10.1016/j.cell.2010.10.009
Conflict of Interest Statement: The authors declare that the research was conducted in the absence of any commercial or financial relationships that could be construed as a potential conflict of interest.

Copyright $\odot 2017$ Hohol, Baran, Krzyżowski and Francikowski. This is an openaccess article distributed under the terms of the Creative Commons Attribution License (CC BY). The use, distribution or reproduction in other forums is permitted, provided the original author(s) or licensor are credited and that the original publication in this journal is cited, in accordance with accepted academic practice. No use, distribution or reproduction is permitted which does not comply with these terms. 